\title{
Change in the hormone receptor status following administration of neoadjuvant chemotherapy and its impact on the long-term outcome in patients with primary breast cancer
}

\author{
T Hirata', C Shimizu*,', K Yonemori', A Hirakawa², T Kouno', K Tamura', M Ando', N Katsumata' and \\ Y Fujiwara'
}

'Department of Breast and Medical Oncology, National Cancer Center Hospital, Chuo-ku, Tokyo 104-0045, Japan; ${ }^{2}$ Department of Management Science, Graduate School of Engineering, Tokyo University of Science, Shinjuku-ku, Tokyo I62-860 I, Japan

\begin{abstract}
BACKGROUND: To evaluate the impact of change in the hormone receptor (HR) status (HR status conversion) on the long-term outcomes of breast cancer patients treated with neoadjuvant chemotherapy (NAC).

METHODS: We investigated 368 patients for the HR status of their lesions before and after NAC. On the basis of the HR status and the use/non-use of endocrine therapy (ET), the patients were categorised into four groups: Group A, I 84 ET-administered patients with HR-positive both before and after NAC; Group B, 47 ET-administered patients with HR status conversion; Group C, 12 ET-naive patients with HR status conversion; Group D, 125 patients with HR-negative both before and after NAC.

RESULTS: Disease-free survival in Group B was similar to that in Group A (hazard ratio, 1.16; $P=0.652$ ), but that in Group C was significantly lesser than that in Group A (hazard ratio, 6.88; $P<0.001$ ). A similar pattern of results was obtained for overall survival. CONCLUSION: Our results indicate that the HR status of tumours is a predictive factor for disease-free and overall survival and that ET appears to be suitable for patients with HR status conversion. Therefore, both the CNB and surgical specimens should be monitored for HR status.
\end{abstract}

British Journal of Cancer (2009) I 0 I, I529- 1536. doi:I0.1038/sj.bjc.6605360 www.bjcancer.com

Published online 6 October 2009

(c) 2009 Cancer Research UK

Keywords: breast cancer; endocrine therapy; hormone receptor status change; neoadjuvant chemotherapy; prognosis

Neoadjuvant chemotherapy (NAC) was introduced in the early 1980 s for patients with locally advanced breast cancer, initially to improve the operability of tumours (Kaufmann et al, 2003). Recently, the application of this therapy has been extended to cases of operable disease. The previously reported results of a metaanalysis indicated that neoadjuvant and adjuvant chemotherapy are equivalent in terms of overall survival (OS) and disease-free survival (DFS) (Mauri et al, 2005). As the pertinent published reports present conflicting views, the actual indications for NAC remain controversial.

Before the initiation of NAC, core-needle biopsy (CNB) is often performed to establish the histological diagnosis and to assess certain factors considered predictive of treatment outcomes. The hormone receptor (HR) status is one such factor. Although this status is known to change after NAC (Bottini et al, 1996; Lee et al, 2003; Taucher et al, 2003; Colleoni et al, 2004; Burcombe et al, 2005; Shet et al, 2007; Tacca et al, 2007; Kasami et al, 2008; Neubauer et al, 2008), its impact on long-term outcomes has not been assessed. The objective of this retrospective study was to

*Correspondence: Dr C Shimizu, Department of Breast and Medical Oncology, National Cancer Center Hospital, 5-I-I Tsukiji, Chuo-ku, Tokyo 104-0045, Japan; E-mail: cshimizu@ncc.go.jp

Received 26 May 2009; revised 7 September 2009; accepted 10 September 2009; published online 6 October 2009 evaluate the frequency and impact of change in the HR status (HR status conversion) on the long-term outcomes in the NAC-administered breast cancer patients.

\section{MATERIALS AND METHODS}

\section{Patients}

We selected 459 primary breast cancer patients treated at the National Cancer Center Hospital between May 1995 and July 2007. All the patients had received anthracycline- and taxane-based NAC. The clinical stages of the patients ranged from cT2N0M0 to cT4dN3M0, which includes inflammatory (T4d) carcinoma. Data were collected on the pre- and post-NAC statuses of oestrogen receptor (ER), progesterone receptor (PgR), and human epidermal receptor (HER) 2 expressions in the lesions. Patients in whom pathologic complete response (pCR) was obtained (91 patients) after surgery, including those with only residual ductal carcinoma in situ (DCIS), were excluded from this analysis because the HR status of the lesions of these patients could not be accurately evaluated. The remaining 368 patients were classified into four groups on the basis of the HR status of their lesions before and after NAC and the use/non-use of endocrine therapy (ET): Group A, 184 ET-administered patients with lesions that were HR-positive 
both before and after NAC; Group B, 47 ET-administered patients with lesions showing HR status conversion; Group C, 12 ET-naive patients with lesions showing HR status conversion; Group D, 125 patients with lesions that were HR-negative both before and after NAC. The mean age at the time of diagnosis of breast cancer was almost the same in the four groups.

\section{Hormone status and HER2 status determination}

All the patients underwent $\mathrm{CNB}$ performed using an $18 \mathrm{G}$ needle. The ER, PgR, and HER2 statuses of all the CNB and surgical specimens were determined by immunohistochemistry (IHC). Details regarding the antibodies used, the clones used, and the time periods for which they were used, as well as the antigen retrieval and the source of antibodies for IHC studies, are listed in Table 1. Positive staining for ER/PgR was defined as nuclear staining in $\geqslant 10 \%$ of the tumour cells. HER2 protein overexpression was defined as with $3+$ complete membrane staining. If HER2 staining on IHC was determined to be $2+$, fluorescent in situ hybridisation (FISH) was used to confirm the results. FISH was performed using the PathVysion kit (Abott-Vysis Lab, Abott Park, IL, USA). HER2 gene amplification was defined as a HER2:chromosome 17 ratio of $\geqslant 2.1$. HR positivity was defined as positivity for ER and/or PgR. The Allred scoring system was used to assess the degree of staining (Allred et al, 1998). Standard controls were prepared on a daily basis for each tumour to ensure the results of IHC.

\section{Tumour size determination and evaluation of neoadjuvant chemotherapy response}

Before each chemotherapy treatment and before surgery, the two greatest perpendicular diameters of the tumours in the breast and axillary nodes were measured, and the products of these diameters were added as a measure of total tumour size. No clinical response of palpable tumour in the breast and axillary lymph nodes was defined as a complete response (CR). Reduction in total tumour size of $50 \%$ or greater was graded as a partial response (PR). An increase in total tumour size of more than $50 \%$ or the appearance of new suspicious ipsilateral axillary adenopathy was considered as a progressive disease (PD). Tumours that did not meet the criteria for objective response or progression were considered as a stable disease (SD).

\section{Chemotherapy}

Patients receiving NAC were administered an anthracycline and a taxane, either concurrently or sequentially. Those receiving concurrent therapy were administered four cycles (doxorubicin at $50 \mathrm{mg} \mathrm{m}^{-2}$ plus docetaxel at $60 \mathrm{mg} \mathrm{m}^{-2}$ ) every 21 days. Patients showing clinical $\mathrm{CR}$ or $\mathrm{PR}$ to the above treatment were administered two additional cycles of the same regimen after the surgery. However, patients who did not achieve objective clinical response to NAC were administered with four cycles of 5-fluorouracil $\left(600 \mathrm{mg} \mathrm{m}^{-2}\right)$, methotrexate $\left(40 \mathrm{mg} \mathrm{m}^{-2}\right)$, and cyclophosphamide $\left(600 \mathrm{mg} \mathrm{m}^{-2}\right)$ after the surgery. For patients receiving the sequential regimen, four cycles of 5-fluorouracil $\left(500 \mathrm{mg} \mathrm{m}^{-2}\right)$, epirubicin $\left(100 \mathrm{mg} \mathrm{m}^{-2}\right)$, cyclophosphamide $\left(500 \mathrm{mg} \mathrm{m}^{-2}\right.$ ) or doxorubicin $\left(60 \mathrm{mg} \mathrm{m}^{-2}\right)$, and cyclophosphamide $\left(600 \mathrm{mg} \mathrm{m}^{-2}\right)$ were administered every 21 days, followed by a taxane. As a taxane, paclitaxel was administered weekly at a dose of $80 \mathrm{mg} \mathrm{m}^{-2}$ per week for 12 weeks or at a dose of $175 \mathrm{mg} \mathrm{m}^{-2}$ every 3 weeks for four cycles, or docetaxel was administered every 3 weeks at a dose of $75 \mathrm{mg} \mathrm{m}^{-2}$ for four cycles.

\section{Adjuvant endocrine therapy (ET) and irradiation}

Adjuvant radiotherapy was administered to patients who underwent breast-conserving surgery. Adjuvant radiotherapy was recommended to those who underwent modified radical mastectomy for the disease ranging from cT3N1M0 to cT4dN3M0. The decision to administer ET was taken on the basis of the treating physician's and/or the patient's preferences. Most patients with HR-positive lesions were administered $20 \mathrm{mg}$ of tamoxifen daily for 5 years. From 2005 onwards, postmenopausal women taking tamoxifen were (1) allowed to switch to an aromatase inhibitor before completing 5 years of tamoxifen, (2) allowed to begin taking an aromatase inhibitor after a 5-year course of tamoxifen or (3) recommended an aromatase inhibitor for the first 5 years.

\section{Statistical analysis}

The frequencies and descriptive statistics of the demographic and clinical variables from the four groups-A, B, C, and D-were obtained. The ER and PgR statuses of the lesions before and after NAC were compared using the consistency test. DFS was defined as the time from surgery to the detection of relapse, death from any cause, or the date of the last visit for patients without events. OS was defined as the time from surgery to death from any cause or the date of the last visit for patients without events. DFS and OS were estimated using the Kaplan-Meier method, and the survival curves were compared using the log-rank test. Multivariate Cox regression analysis with stepwise selection $(\alpha=0.05)$ was used to estimate the hazard ratio, $95 \%$ confidence interval (CI), and the effects of the clinical and pathological variables. A two-sided $P<0.05$ was considered to be statistically significant. All the analyses were performed using the SAS (version 9.1; SAS Institute Inc., Cary, NC, USA).

Table I Panel of antibodies

\begin{tabular}{|c|c|c|c|c|c|}
\hline Antigen & Period used for & Clone & Type & Antigen retrieval & Source \\
\hline \multirow[t]{3}{*}{ ER } & Until Oct 2002 & ID5 & Mouse monoclonal & AVC citrate buffer & Dako \\
\hline & From Nov 2002 to Feb 2005 & ER88 & Mouse monoclonal & As above & Bio Genex \\
\hline & From Mar 2005 & ID5 & Mouse monoclonal & As above & Dako \\
\hline \multirow{3}{*}{$\mathrm{PgR}$} & Until Oct 2002 & IA6 & Mouse monoclonal & As above & Novocastra \\
\hline & From Nov 2002 to Feb 2005 & PR88 & Mouse monoclonal & As above & Bio Genex \\
\hline & From Mar 2005 & PgR636 & Mouse monoclonal & As above & Dako \\
\hline \multirow[t]{3}{*}{ HER2 } & Until Oct 2002 & c-erbB-2 & Rabbit polyclonal & As above & Dako \\
\hline & From Nov 2002 to Feb 2005 & CBII & Mouse monoclonal & As above & Bio Genex \\
\hline & From Mar 2005 & c-erbB-2 & Rabbit polyclonal & As above & Dako \\
\hline
\end{tabular}

Abbreviations: A/C: autoclave for $10 \mathrm{~min}$ at $121^{\circ} \mathrm{C}$; ER: estrogen receptor; HER2: human epidermal receptor 2; PgR: progesterone receptor; citrate buffer: 10 mM citrate buffer, $\mathrm{pH} 6.0$. 
Table 2 Patient and tumour characteristics

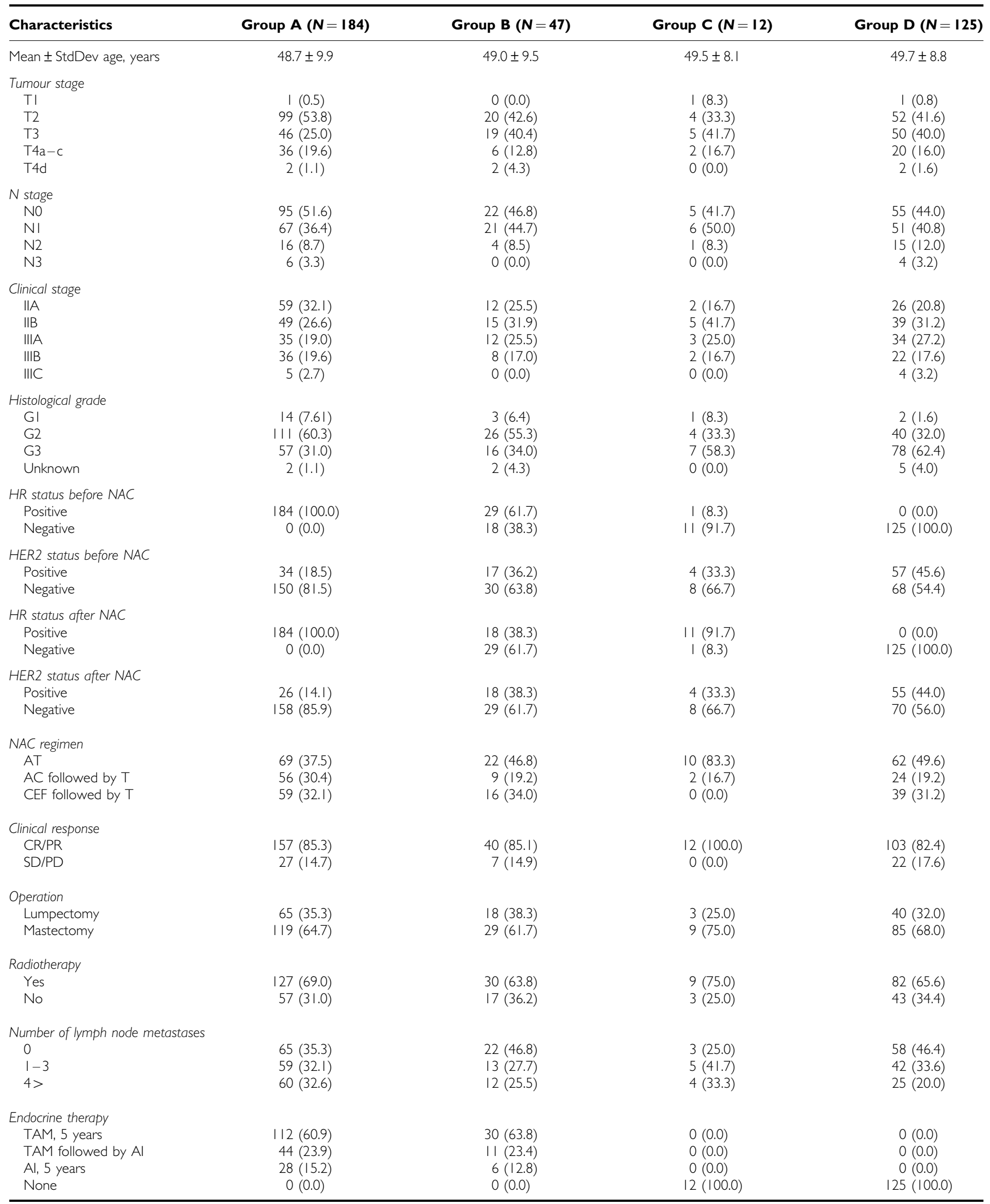

Abbreviations: $\mathrm{AC}=$ doxorubicin and cyclophosphamide; $\mathrm{Al}=$ aromatase inhibitor; $\mathrm{AT}=$ doxorubicin and docetaxel; $\mathrm{CEF}=$ cyclophosphamide, epirubicin and 5-fluorouracil; $\mathrm{ER}=$ estrogen receptor; HER2 = human epidermal receptor; $N=$ number of patients; PgR= progesterone receptor; $T=$ taxane (weekly or triweekly paclitaxel, or triweekly docetaxel); TAM = tamoxifen; Figures in parentheses are percentage of patients except for age. 

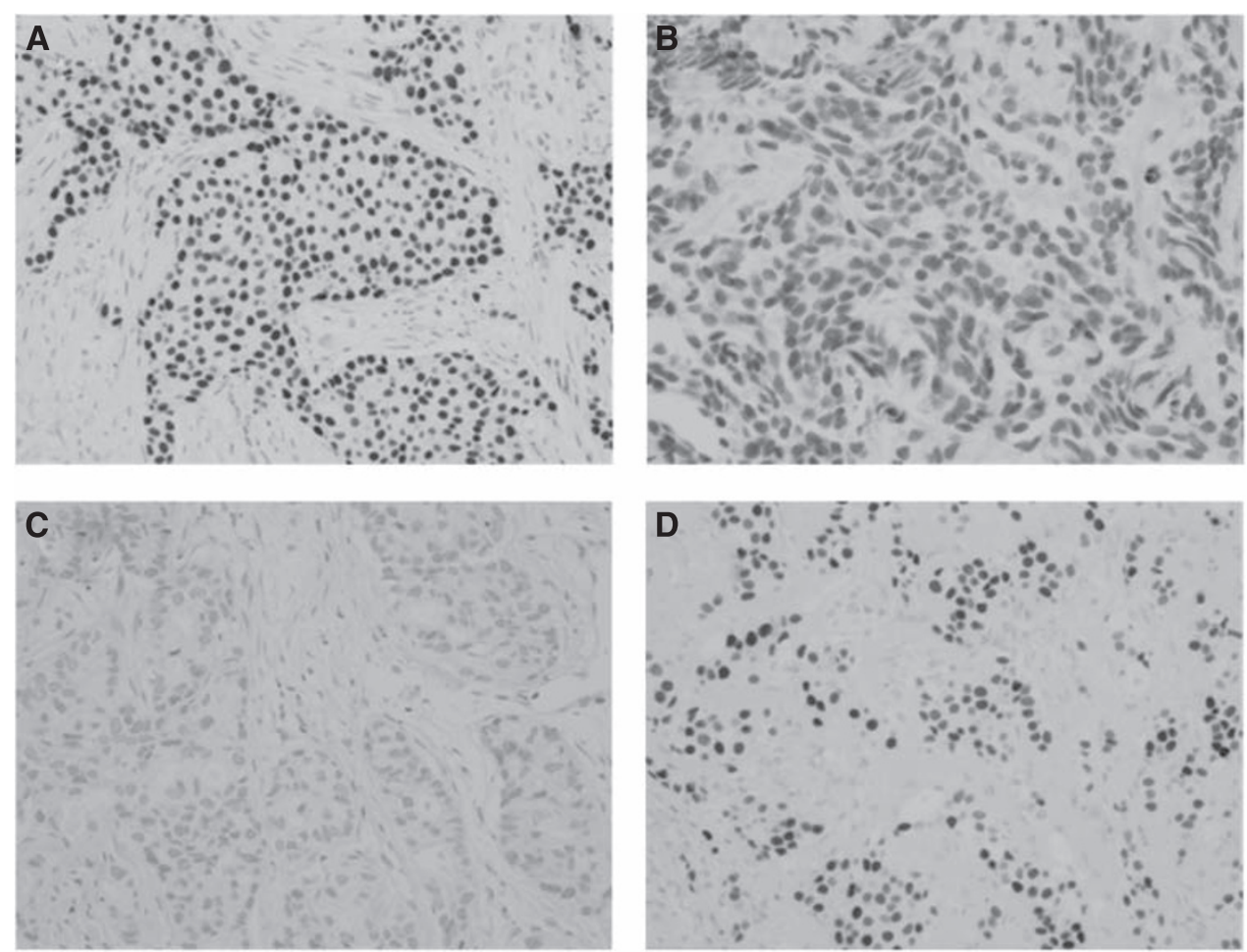

Figure I Immunostaining for oestrogen receptor in core needle biopsy and surgery specimens after neoadjuvant chemotherapy. (A) Staining of tumour cells in core-needle biopsy sample (CNB) staining positively for oestrogen receptor (ER). (B) Staining of tumour cells in surgical samples with ER-negative status after neoadjuvant chemotherapy (NAC). (C) Staining of tumour cells in CNB specimens with ER-negative status. (D) Staining of tumour cells in surgical samples with ER-positive status after NAC.

\section{RESULTS}

\section{Patient characteristics}

Among the 459 NAC-administered patients, pCR after surgery was achieved in 91 patients. Pathological assessment of the CNB specimens of patients with pCR revealed that $26(28.6 \%)$ and 19 (20.9\%) patients, respectively, were ER-positive and PgR-positive and that $63(69.2 \%)$ were negative for both ER and PgR.

Examination of the surgical specimens revealed residual invasive disease in 368 patients. The distribution of these patients in the four groups was as follows: Group A, 184 (50.0\%) patients; Group B, 47 (12.8\%) patients; Group C, 12 (3.3\%) patients; Group $\mathrm{D}, 125(34.0 \%)$ patients.

The patient and tumour characteristics of the four groups are listed in Table 2. The postoperative performance status (PS 0 or 1) of all the patients was good. HR status conversion after NAC was observed in $59(16.0 \%)$ patients. None of the HER2-positive patients were administered trastuzumab during neoadjuvant or adjuvant chemotherapy. Twelve $(3.3 \%)$ did not receive adjuvant ET, although it was not contraindicated, but all of their lesions showed HR status conversion after NAC. All patients whose lesions showed ER status from positive to negative after chemotherapy had been administered ET.

\section{Change in the HR status and HER2 status}

The typical staining patterns of the CNB and surgical specimens are shown in Figure 1. The pre- and post-NAC ER and PgR statuses are shown in Table 3. Lesions of $23(6.3 \%)$ patients showed a change in both the ER and PgR statuses after NAC. The HR and HER2 statuses changed from positive to negative in $30(8.2 \%)$ and $22(6.0 \%)$ patients, and changed from negative to positive in 29 (7.9\%) and $13(3.5 \%)$ patients, respectively.
Table 3 Number of patients classified by estrogen receptor and progesterone receptor statuses before and after neoadjuvant chemotherapy

(ER, PgR) after NAC

\begin{tabular}{lccrr}
$($ ER, PgR) before NAC & $\mathbf{( + , ~ + )}$ & $\mathbf{( + , ~})$ & $(-,+)$ & $(-,,-)$ \\
\hline$(+,+)$ & $69(18.8)$ & $41(11.1)$ & $3(0.8)$ & $10(2.7)$ \\
$(+,-)$ & $18(4.9)$ & $27(7.3)$ & $1(0.3)$ & $7(1.9)$ \\
$(-,+)$ & $11(3.0)$ & $6(1.6)$ & $8(2.2)$ & $13(3.5)$ \\
$(-,-)$ & $6(1.6)$ & $11(3.0)$ & $12(3.3)$ & $125(34.0)$ \\
\hline
\end{tabular}

Abbreviations: $\quad E R=$ estrogen receptor; $H E R 2=$ human epidermal receptor 2; $\mathrm{PgR}=$ progesterone receptor. Figures in parentheses are percentage of patients.

Figures 2 and 3 show the pre- and post-NAC proportion, intensity, and total scores of ER and PgR staining, determined on the basis of the Allred scoring system, for patients who underwent a change in the HR status. As shown in Figures 2 and 3, the changes in the ER and PgR statuses were observed not only in cases with borderline positive (total score $3-5$ ) staining but also in those with strongly positive (total score 7-8) staining. The change in the HR status was not caused by a change in only the proportion or intensity scores of ER and PgR. In addition, Figure 4 shows the results of HER2 testing in the 59 patients who showed HR status conversion.

\section{Long-term outcomes}

The median duration of follow-up was 47 months. Figure 5 shows the Kaplan-Meier curves for DFS in the four groups. The differences among the four curves were statistically significant, 
A

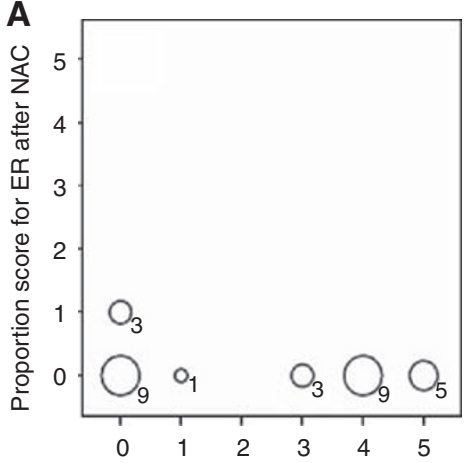

Proportion score for ER before NAC

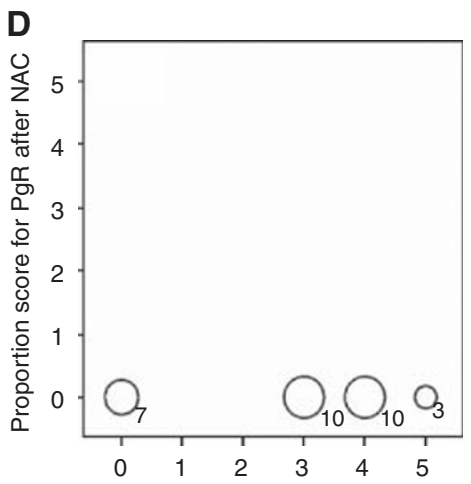

Proportion score for PgR before NAC

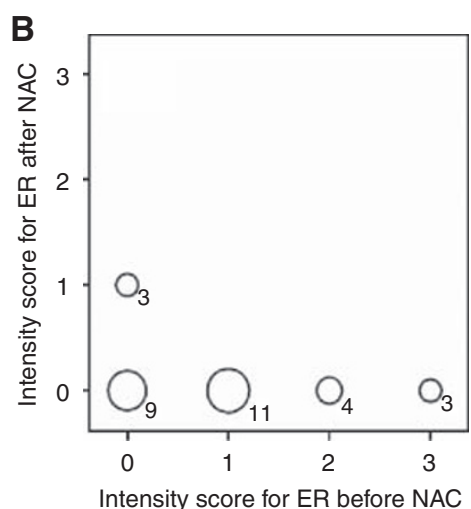

E

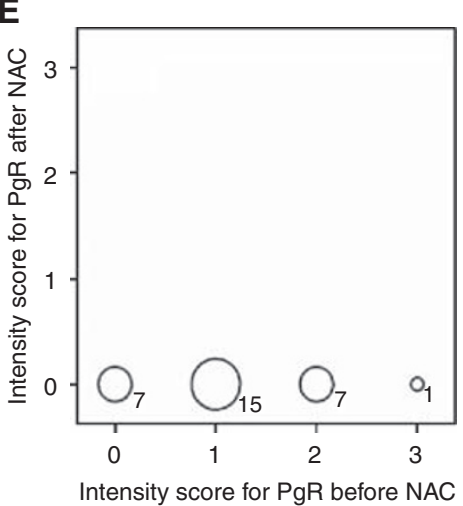

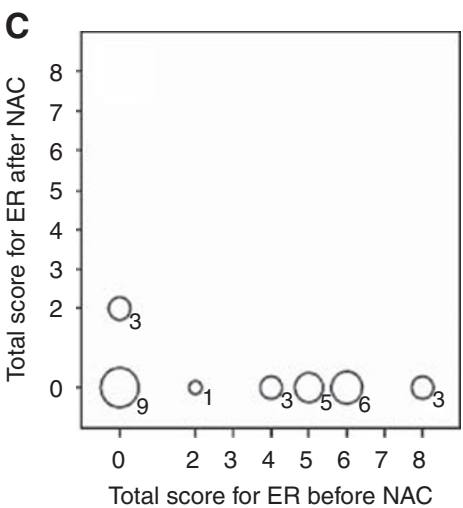

$\mathbf{F}$

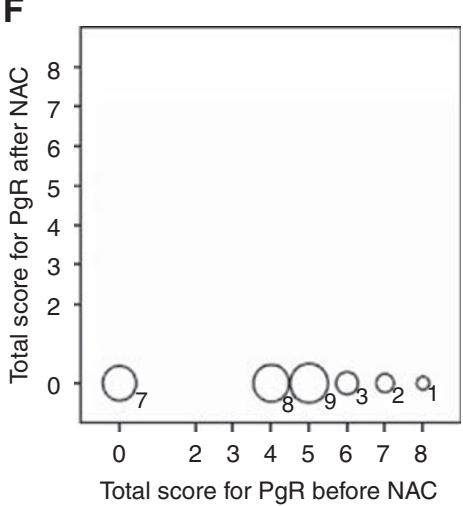

Figure 2 The distribution of scores for oestrogen receptor and progesterone receptor staining before and after neoadjuvant chemotherapy in 30 patients whose lesions changed from hormone receptor (HR)-positive status to HR-negative status. The size of the circle indicates the number of patients and the number is below the circle. (A-C) Proportion score, intensity score and total score of ER before and after NAC. (D-F) Proportion score, intensity score and total score of PgR befoe and after NAC.

as determined by the log-rank test $(P=0.008)$. The 3-year DFS rates in Groups A, B, C, and D were 80.3, 78.4, 36.4, and $72.2 \%$, respectively.

Table 4 shows the results of the multivariate Cox regression analysis of DFS with stepwise selection. The following six variables were chosen as prognostic factors for inclusion in the Cox proportional hazard model: age ( $<35 v s \geqslant 35$ years), clinical stage at diagnosis (IIA and IIB, or IIIA $v s$ IIIB or IIIC), histological grade ( 1 vs 2 and 3), HER2 status (positive $v s$ negative), clinical response (CR, PR vs SD, PD), and the number of lymph node metastases $(0 v s 1-3 v s \geqslant 4)$. Three of these variables-the HER2 status, clinical response to NAC, and the number of lymph node metastases-were identified by the stepwise selection method in the multivariate Cox regression model as the variables affecting the DFS.

The DFS of Groups B and A was similar (hazard ratio, 1.16; 95\% CI, 0.61-2.19), whereas that of Group C was significantly shorter than that of Group A (hazard ratio, 6.88; 95\% CI, 3.00-15.80). Table 5 summarises the results of the analysis of the efficacy of ET in the 59 patients who showed HR status conversion by using the multivariate Cox regression model. The DFS of the ET-administered patients was significantly longer than that of ET-naive patients (hazard ratio, $0.19 ; 95 \%$ CI, $0.06-0.60 ; P<0.004$ ).

Figure 6 shows the Kaplan-Meier curves for OS in the four groups. The differences among the four curves were statistically significant, as determined by the log-rank test $(P=0.035)$. The 5-year survival rates of Groups A, B, C, and D were 90.3, 86.3, 58.9, and $78.2 \%$, respectively. The pattern of results of the analyses for OS in the four groups was similar to that for DFS.

\section{DISCUSSION}

This is the first report on the long-term outcomes and impact of adjuvant ET in patients with HR status conversion after NAC. In this study, the DFS and the OS of ET-administered patients with HR status-converted lesions were similar to those of ET-administered patients with lesions that were HR-positive both before and after NAC, whereas the DFS of ET-naive patients whose lesions show HR status conversion was significantly shorter than that of ET-administered patients whose lesions were HR-positive both before and after NAC. Analysis of OS yielded results similar to that pertaining to DFS. These findings indicate that the change in the status alone did not seem to influence the long-term outcome; rather, the non-administration of adjuvant ET seemed to be associated with a worse prognosis.

ER, PgR, and HER2 status changes were observed in 14.9, 29.1, and $9.5 \%$ of the patients included in our study. The overall frequency of patients with HR status conversion was $16.0 \%$. This incidence of HR status conversion was similar to previous reports on post-NAC change in the ER, PgR, and HER2 statuses, which reported incidences of 8-28\%, 6-59\% (Bottini et al, 1996; Lee et al, 2003; Taucher et al, 2003; Colleoni et al, 2004; Burcombe et al, 2005; Shet et al, 2007; Kasami et al, 2008; Neubauer et al, 2008), and 0-21\% (Bottini et al, 1996; Colleoni et al, 2004; Arens et al, 2005; Burcombe et al, 2005; Quddus et al, 2005; Adams et al, 2008; Kasami et al, 2008; Neubauer et al, 2008), respectively. Although the rate of cases with no change in the HR status after NAC was high, the incidence of change in the HR status is 
A
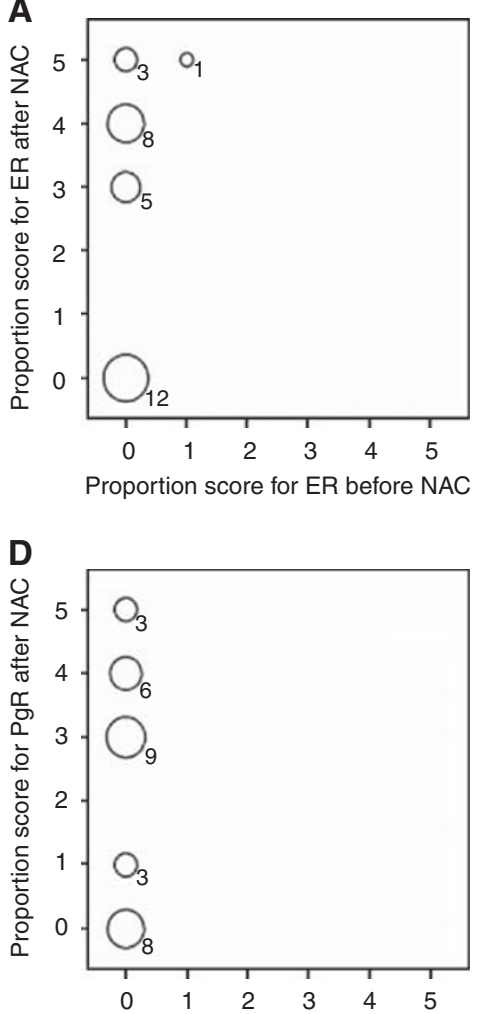

Proportion score for PgR before NAC
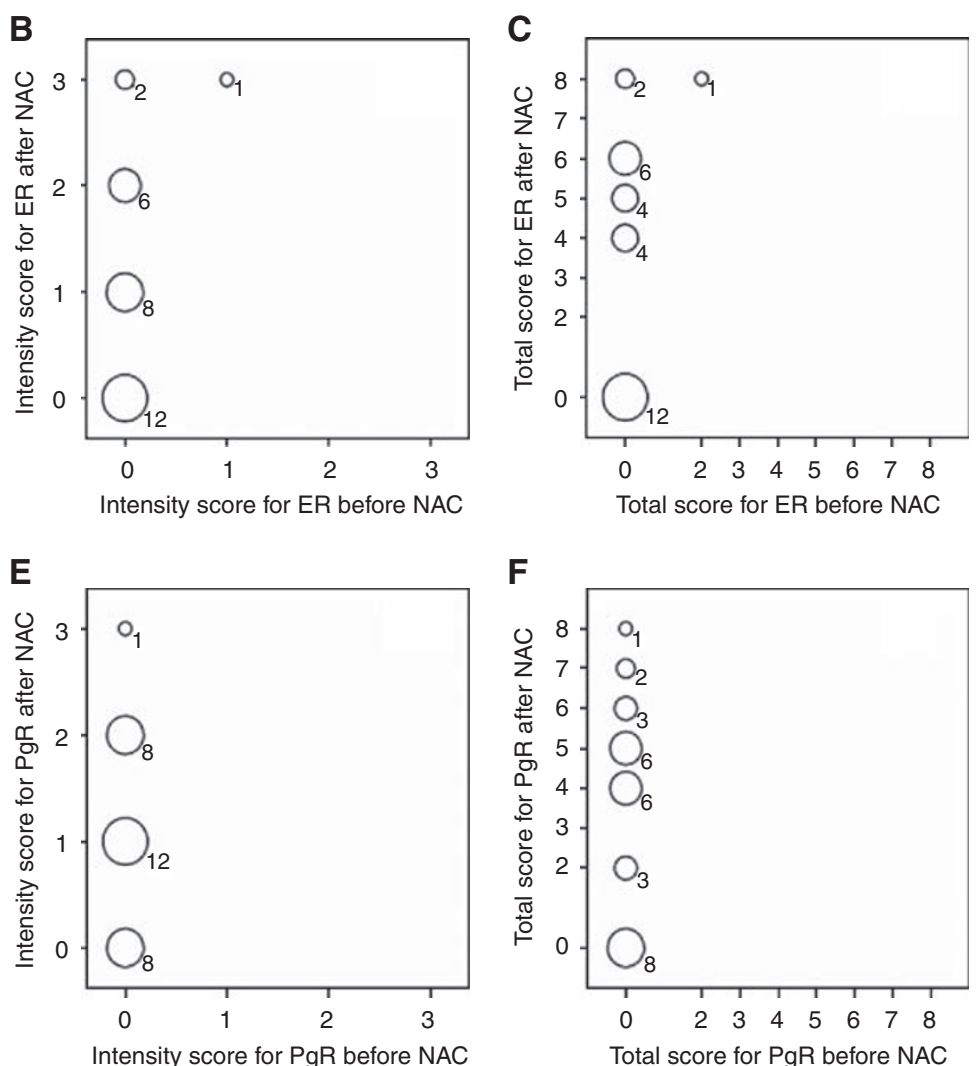

$\mathbf{F}$

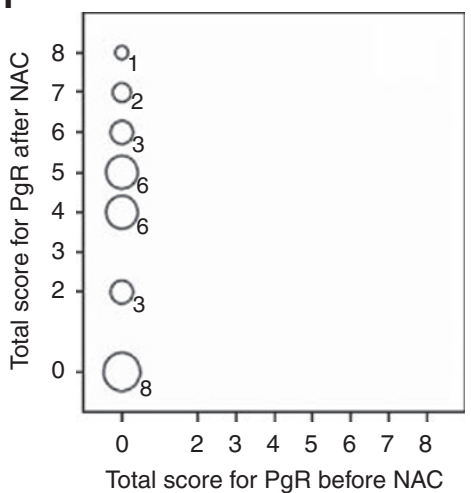

Figure 3 Scores of staining for oestrogen receptor and progesterone receptor before and after neoadjuvant chemotherapy in 29 patients whose lesions changed from being hormone receptor (HR)-negative to HR-positive. The size of the circle indicates the number of patients and the number is below the circle. (A-C) Proportion score, intensity score, total score of ER before and after NAC. (D-F) Proportion score, intensity score, total score of PgR before and after NAC.

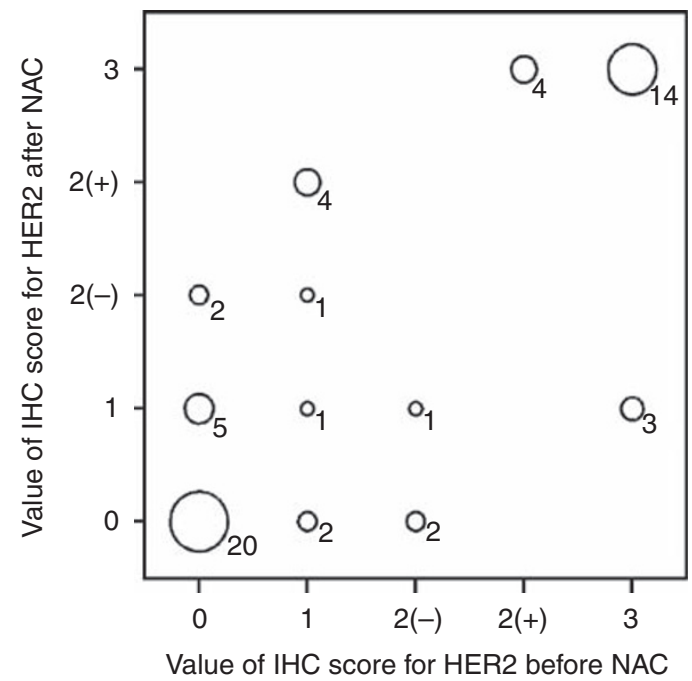

Figure 4 Bubble plot for immunohistochemistry score for HER2 before and after neoadjuvant chemotherapy in 59 patients with hormone receptor status conversion. The figures added to the bubbles are the number of patients and each bubble's size is determined by the number of patients in the category: the more the patients, the larger the bubble. The symbols $(+)$ and $(-)$, respectively, indicate the positive and negative status by fluorescent in situ hybridisation (FISH)

clinically not negligible. The poor prognosis of patients with HR status conversion not administered adjuvant ET indicates the necessity to determine the HR status of the lesions both before and

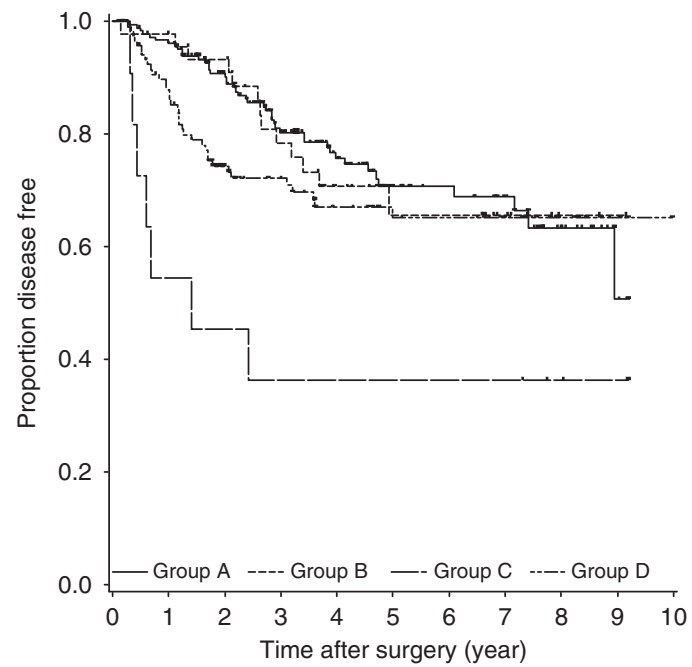

Figure 5 Kaplan-Meier curves of disease-free survival in four groups. Short vertical lines indicate censored data points. Log-rank test was significant for disease-free survival (DFS) $(P=0.008)$.

after NAC and to administer ET to patients with HR status conversion.

Despite yielding these clinically relevant findings, our study is limited in some aspects: (1) The patient groups studied were heterogeneous in terms of sample size and characteristics. (2) This study was retrospective and the results of the statistical tests were not based on randomisation, but were exploratory, although the 
Table 4 Results of multivariate Cox regression analysis of disease-free survival with stepwise selection

\begin{tabular}{|c|c|c|}
\hline Variables & Hazard ratio $(95 \% \mathrm{Cl})$ & $P$-value \\
\hline \multicolumn{3}{|l|}{ Group } \\
\hline A & 1 & \\
\hline B & $1.16(0.61,2.19)$ & 0.652 \\
\hline C & $6.88(3.00,15.80)$ & $<0.001$ \\
\hline $\mathrm{D}$ & $1.63(1.01,2.63)$ & 0.045 \\
\hline \multicolumn{3}{|l|}{ Clinical stage } \\
\hline$\|\mathrm{A} /\| \mathrm{B} / \| \mathrm{A}$ & 1 & \\
\hline$\| I B / I I C$ & $1.56(1.00,2.42)$ & 0.049 \\
\hline \multicolumn{3}{|l|}{ HER2 } \\
\hline Negative & I & \\
\hline Positive & $2.00(1.30,3.09)$ & 0.002 \\
\hline \multicolumn{3}{|c|}{ Clinical response } \\
\hline $\mathrm{SD} / \mathrm{PD}$ & 1 & \\
\hline $\mathrm{PR} / \mathrm{CR}$ & $0.56(0.34,0.92)$ & 0.021 \\
\hline \multicolumn{3}{|c|}{ Number of lymph node metastases } \\
\hline 0 & I & \\
\hline $1-3$ & $2.09(1.14,3.83)$ & 0.017 \\
\hline$>4$ & $6.49(3.71,11.37)$ & $<0.001$ \\
\hline
\end{tabular}

Abbreviations: $\mathrm{Cl}=$ confidence interval; $\mathrm{CR}=$ complete response; $\mathrm{PR}=$ partial response; $\mathrm{SD}=$ stable disease; $\mathrm{PD}=$ progression disease.

Table 5 Efficacy of endocrine therapy in patients with lesions showing hormone receptor status conversion after neoadjuvant chemotherapy in terms of disease-free survival

\begin{tabular}{|c|c|c|}
\hline Variables & Hazard ratio $(95 \% \mathrm{Cl})$ & $P$-value \\
\hline \multicolumn{3}{|l|}{ ET } \\
\hline No & 1 & \\
\hline Yes & $0.19(0.06,0.60)$ & 0.004 \\
\hline \multicolumn{3}{|l|}{ HER2 } \\
\hline Negative & 1 & \\
\hline Positive & $1.58(0.46,5.42)$ & 0.467 \\
\hline \multicolumn{3}{|c|}{ Clinical response } \\
\hline $\mathrm{SD} / \mathrm{PD}$ & 1 & \\
\hline $\mathrm{PR} / \mathrm{CR}$ & $0.75(0.15,3.93)$ & 0.738 \\
\hline \multicolumn{3}{|l|}{ Clinical stage } \\
\hline$\|\mathrm{A} /\| \mathrm{B} / \| \mathrm{A}$ & 1 & \\
\hline$\|\mathrm{B} /\| \mathrm{IC}$ & $1.03(0.26,4.16)$ & 0.968 \\
\hline \multicolumn{3}{|c|}{ Number of lymph node metastases } \\
\hline 0 & 1 & \\
\hline $1-3$ & $2.74(0.63,11.98)$ & 0.181 \\
\hline$>4$ & $14.66(3.24,66.43)$ & 0.001 \\
\hline
\end{tabular}

Abbreviations: $\mathrm{Cl}=$ confidence interval; $\mathrm{CR}=$ complete response; $\mathrm{PR}=$ partial response; $\mathrm{SD}=$ stable disease; $\mathrm{PD}=$ progression disease

prognostic factors were adjusted using multivariate Cox regression analysis. Therefore, the impact of the change in the pre- and postNAC HR statuses on the long-term outcomes and the efficacy of ET for patients with HR status conversion should be evaluated using a prospective study design. (3) The methods for measuring the ER

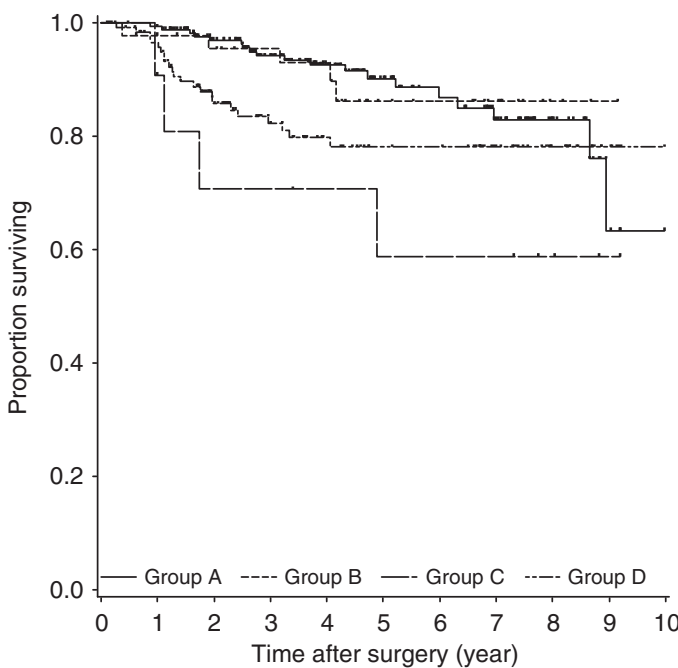

Figure 6 Kaplan-Meier curves of OS in four groups. Short vertical lines indicate censored data points. Log-rank test was significant for overall survival $(O S)(P=0.035)$.

and PgR status varied with the age of the patients, as shown in Table 1. Although the methods used for the determination of the HR statuses of the tumours of 36 patients among 368 patients were measured using different methods for the $\mathrm{CNB}$ and surgical specimens, only three of these tumours showed HR status conversion. A previous report showed that the HR status conversion occurred in $23 \%$ of the population in a study in which the same methods were used for the analysis of $\mathrm{CNB}$ and surgical specimens (Tacca et al, 2007), whereas HR status conversion was observed in $16.0 \%$ (59 patients) of the patients in this study. Therefore, the difference in the methods for measuring the ER and PgR statuses of the CNB and surgical specimens seems not to be the only reason for HR-status conversion.

In conclusion, our study showed that the prognosis of patients with change in HR status after NAC but who did not receive ET was worse than that of the other groups. The hormone receptor status should be evaluated not only in the biopsy specimens obtained before the initiation of NAC but also in specimens obtained during post-NAC surgery; the pre- and post-NAC HR statuses will help determine the indication for adjuvant ET in patients. ET appears to be suitable for patients with tumours positive for HR status at least once, that is, either before or after NAC.

\section{ACKNOWLEDGEMENTS}

We thank Dr Yuko Sasajima and Dr Hitoshi Tsuda of the Department of Pathology, National Cancer Center Hospital, for help with the pathological review. This study was supported by a grant from the Ministry of Health, Labour and Welfare (H21-4-4).

\section{Conflict of interest}

The authors declare that there are no competing financial interests.

\section{REFERENCES}

Adams AL, Eltoum I, Krontiras H, Wang W, Chhieng DC (2008) The effect of neoadjuvant chemotherapy on histologic grade, hormone receptor status, and Her2/neu status in breast carcinoma. Breast J 14: 141 - 146
Allred DC, Harvey JM, Berardo M, Clark GM (1998) Prognostic and predictive factors in breast cancer by immunohistochemical analysis. Mod Pathol 11: $155-168$ 
Arens N, Bleyl U, Hildenbrand R (2005) HER2/neu, p53, Ki67, and hormone receptors do not change during neoadjuvant chemotherapy in breast cancer. Virchows Arch 446: 489-496

Bottini A, Berruti A, Bersiga A, Brunelli A, Brizzi MP, Marco BD, Cirillo F, Bolsi G, Bertoli G, Alquati P, Dogliotti L (1996) Effect of neoadjuvant chemotherapy on Ki67 labelling index, c-erbB-2 expression and steroid hormone receptor status in human breast tumours. Anticancer Res 16: $3105-3110$

Burcombe RJ, Makris A, Richman PI, Daley FM, Noble S, Pittam M, Wright D, Allen SA, Dove J, Wilson GD (2005) Evaluation of ER, PgR, HER-2 and $\mathrm{Ki}-67$ as predictors of response to neoadjuvant anthracycline chemotherapy for operable breast cancer. Br J Cancer 92: 147 - 155

Colleoni M, Viale G, Zahrieh D, Pruneri G, Gentilini O, Veronesi P, Gelber RD, Curigliano G, Torrisi R, Luini A, Intra M, Galimberti V, Renne G, Nolè F, Peruzzotti G, Goldhirsch A (2004) Chemotherapy is more effective in patients with breast cancer not expressing steroid hormone receptors: a study of preoperative treatment. Clin Cancer Res 10: 6622-6628

Kasami M, Uematsu T, Honda M, Yabuzaki T, Sanuki J, Uchida Y, Sugimura H (2008) Comparison of estrogen receptor, progesterone receptor and Her-2 status in breast cancer pre- and post-neoadjuvant chemotherapy. Breast 17: 523-527

Kaufmann M, von Minckwitz G, Smith R, Valero V, Gianni L, Eiermann W, Howell A, Costa SD, Beuzeboc P, Untch M, Blohmer JU, Sinn HP, Sittek R, Souchon R, Tulusan AH, Volm T, Senn HJ (2003) International expert panel on the use of primary (preoperative) systemic treatment of operable breast cancer: review and recommendations. J Clin Oncol 21: $2600-2608$
Lee SH, Chung MA, Quddus MR, Steinhoff MM, Cady B (2003) The effect of neoadjuvant chemotherapy on estrogen and progesterone receptor expression and hormone receptor status in breast cancer. Am J Surg 186: $348-350$

Mauri D, Pavlidis N, Ioannidis JP (2005) Neoadjuvant versus adjuvant systemic treatment in breast cancer: meta-analysis. J Natl Cancer Inst 97: $188-194$

Neubauer H, Gall C, Vogel U, Hornung R, Wallwiener D, Solomayer E, Fehm T (2008) Changes in tumour biological markers during primary systemic chemotherapy (PST). Anticancer Res 28: 1797-1804

Quddus RM, Sung JC, Zhang C, Pasqueriello T, Eklund M, Steinhoff MM (2005) HER-2/neu expression in locally advanced breast carcinomas: Pre-and post-neoadjuvant chemotherapy. Breast Cancer 12: 294-298

Shet T, Agrawal A, Chinoy R, Havaldar R, Parmar V, Badwe R (2007) Changes in the tumor grade and biological markers in locally advanced breast cancer after chemotherapy-implications for a pathologist. Breast $J$ 13: $457-464$

Tacca O, Penault-Llorca F, Abrial C, Mouret-Reynier MA, Raoelfils I, Durando X, Achard JL, Gimbergues P, Curé H, Chollet P (2007) Changes in and prognostic value of hormone receptor status in a series of operable breast cancer patients treated with neoadjuvant chemotherapy. Oncologist 12: 636-643

Taucher S, Rudas M, Gnant M, Thomanek K, Dubsky P, Roka S, Bachleitner T, Kandioler D, Wenzel C, Steger G, Mittlböck M, Jakesz R (2003) Sequential steroid hormone receptor measurements in primary breast cancer with and without intervening primary chemotherapy. Endocr Relat Cancer 10: $91-98$ 